\title{
Motivación de logro y metas en la vida: Rol mediador de la inteligencia emocional
}

\section{Carmen María Salvador-Ferrer ${ }^{1}$}

${ }^{1}$ Departamento de Psicología. Universidad de Almería, Almería.

\section{España}

Correspondencia: Salvdor-Ferrer, Carmen María. Carretera Sacramento. La Cañada de San Urbano, s/n. C.P. 04120. España. E-mail: cmsalva@ual.es

(C) Universidad de Almería and Ilustre Colegio Oficial de la Psicología de Andalucía Oriental (Spain) 


\section{Resumen}

Introducción. Se realiza un estudio ex post facto cuyo objetivo consiste en analizar la relación entre la motivación de logro y la inteligencia emocional en las metas vitales de los estudiantes, para conocer si la inteligencia emocional es una variable mediadora entre la motivación de logro y las metas vitales.

Método. El trabajo se llevó a cabo en una muestra de 311 estudiantes universitarios, en los que se aplicaron las escalas de Trait Meta-Mood Scale (TMMS; Salovey et al., 1995), Escala de motivación de logro (ML; Morales, 2006) y escala de Metas en la vida (Wilding y Andrews, 2006). Se realizó un análisis de mediación, utilizando la macro PROCESS de SPSS, con técnicas brootstrapping (10000 brootstrapping, modelo 4 con un nivel de confianza del $95 \%)$.

Resultados. Los resultados indican que la motivación de logro influye positivamente y significativamente sobre la inteligencia emocional y las metas vitales. Además, encontramos que la relación entre la motivación de logro y las metas vitales se encuentra mediada positivamente y significativamente por la inteligencia emocional.

Discusión y conclusiones. Con estos hallazgos, nuestra investigación podría ofrecer una contribución innovadora ofreciendo un modelo teórico que muestra la relación establecida entre las variables, en el que se aprecia el papel amortiguador que la inteligencia emocional presenta en la influencia de la relación entre la motivación de logro y las metas vitales.

Palabras Clave: Motivación de logro, Metas vida, Inteligencia emocional, Mediación. 


\begin{abstract}
Introduction: This paper seeks to analyse the relationship about achievement motivation, life goals and emotional intelligence. The present study aims to investigate the role of achievement motivation and emotional intelligence in life goals, verifying if emotional intelligence mediates the relationship among achievement motivation and life goals.

Method: In this project had been participated 311 universitary students. The scales used are Trait Meta-Mood Scale (TMMS; Salovey et al., 1995), Achievement motivation scale (ML; Morales, 2006) and Life goals (Wilding y Andrews, 2006). The satidistic analysis were been doing in SPSS. With the macro PROCESS, mediation analyzes were performed with brootstrapping techniques (10000 brootstrapping, model 4 with a confidence level of 95\%).

Results: The results indicate that achievement motivation positively and significantly influences emotional intelligence and life goals. We found that the relationship between achievement motivation and life goals is positively and significantly mediated by emotional intelligence.

Discussion or Conclusion: Our research could offer an innovative contribution by offering a theoretical model that shows the relationship established between the terms, in which the buffering role that emotional intelligence presents in the influence of the relationship between achievement motivation and achievement is appreciated vital goals.
\end{abstract}

Keywords: Achievement motivation, Life Goals, Emotional intelligence, Mediation. 


\section{Introducción}

La inteligencia emocional es una capacidad adquirida que ayuda a determinar la causa que genera nuestra emoción (Salovey y Mayer, 1990; Nathanson et al., 2016) y, de este modo, favorece el ajuste de nuestras emociones al contexto social (Cooper y Sawaf, 1997), por lo que, según señala Goleman (1995), la inteligencia emocional es una competencia básica que dirige nuestros sentimientos y acciones, y, también, ayuda a comprender cómo se sienten otras personas.

La inteligencia emocional, por tanto, orienta nuestras percepciones, sentimientos y acciones en diferentes parcelas vitales (Mayer et al., 2004), por ejemplo, en el ámbito familiar, laboral, académico, etc. Las investigaciones realizadas, entre otros autores, por Gardner (1993) y Pekrun (1992) reflejan la importancia de la gestión emocional en la integración social y, también, para lograr el éxito académico. En el modelo elaborado por Collaborative for Academic, Social, and Emotional Learning -CASEL- (2013) se recoge la relevancia social y académica de la inteligencia emocional, así como los elementos que pueden estar relacionados con ésta. En concreto, en dicho modelo se identifican 5 factores: Conciencia de sí mismo, se trata de la competencia para reconocer las emociones y los pensamientos personales; Autogestión, es el talento para gestionar las emociones y los pensamientos en diferentes situaciones, incluye, entre otros elementos, la motivación de uno mismo para establecer y lograr metas; Conciencia social, disposición para empatizar con otras personas; Habilidades de relación, se trata de la aptitud para establecer relaciones satisfactorias con diferentes personas y grupos. Y, finalmente, la toma de decisiones responsables, que es la habilidad para tomar decisiones positivas, proactivas y respetuosas.

Teniendo como referente este modelo teórico, centramos nuestro estudio en los factores de autogestión y toma de decisiones. En el primer componente analizaremos la motivación de logro, mientras que en el segundo elemento nos centraremos en las metas vitales, ya que, tal como señala Edmund (2011), la toma de decisiones se encuentra impulsada por las convicciones y las metas vitales. En concreto, las metas vitales, junto con otros términos (por ejemplo, los valores, los intereses, habilidades, etc.), facilitan la toma de decisiones académicas y profesionales (Ocampo et al., 2001; Lozano y Repetto, 2007). 
Los hechos fundamentales que nos llevan a estudiar la relación entre estos tres elementos (inteligencia emocional, motivación de logro y metas vitales) son, en primer lugar, el escaso número de investigaciones centradas en estos términos que ofrezcan un modelo teórico causa-efecto. En segundo lugar, los estudios sobre inteligencia emocional se han centrado básicamente en los aspectos cognitivos, no atendiendo los factores motivacionales (García y Domenech, 1997). Sirva como referente el trabajo de Pekrun (1992), quien elabora un marco teórico donde relaciona las emociones y el estado de ánimo con el aprendizaje y rendimiento, es decir, un modelo emotivo-motivacional. Tal como señala este autor los efectos de las emociones en el aprendizaje y el logro se encuentran mediados por una serie de aspectos cognitivos y motivacionales. Por tanto, la relevancia debe ser motivar a los estudiantes para que aprendan estrategias de estudio y de autorregulación del aprendizaje. No obstante, hay que destacar que el nexo entre la motivación de logro y las emociones es complejo, puesto que podría verse afectado por diferentes elementos (Pekrun et al., 2002).

De manera más específica, en el ámbito académico resulta importante estudiar tanto la inteligencia cognitiva como la emocional, ya que ésta puede ser un determinante de la motivación de logro y de la calidad vital estudiantil (Yazici et al., 2011). Pese a todo, podríamos decir que el número de trabajos que valoran la relación entre la inteligencia emocional y la motivación de logro es bastante reducido. La motivación de logro es un elemento que influye en cualquier proceso de enseñanza y aprendizaje (Fernández, 2008). En términos generales, podría definirse como una necesidad recurrente de manejar tareas difíciles para tener éxito (Takeuchi et al., 2014). La motivación de logro permite pronosticar la continuidad académica en la calidad del aprendizaje. Es tal la importancia de este término que se han impulsado algunas investigaciones, poniendo de manifiesto la relación entre la motivación de logro y una amplia gama de situaciones y condiciones, influyendo factores como la dificultad de la tarea y las capacidades individuales (Takeuchi et al., 2014).

En relación con la motivación de logro, encontramos los trabajos de Chowdhury et al. (2007), quienes establecieron que ésta orienta a los estudiantes en el cumplimiento de sus objetivos (Valdés et al., 2013), es decir, la motivación de logro se relaciona con los propósitos o las metas vitales. Según Ryff (1989), entre otros factores, las metas y/o los proyectos personales son fundamentales para tener un sentido vital. Van Damme et al. (2012) proponen la necesidad de estudiar los mecanismos motivacionales que permiten que las personas sean perseverantes en sus acciones, es decir, conocer los mecanismos de auto-regulación de los objetivos 
en el contexto. En esta línea, también, conviene destacar el trabajo de Hardin et al. (2019), quienes descubrieron la existencia de una relación entre la motivación de logro y la permanencia académica.

En otro ángulo, Dulewicz y Higgs (2000) impulsan una investigación sobre la motivación de logro, donde concluyen que este término puede estar relacionado con la inteligencia emocional. Estos autores han demostrado la estrecha concordancia que existe entre la motivación de logro y la inteligencia emocional (Dulewicz y Higgs, 1999, 2000), confirmándose dicha relación en otros estudios (Roy et al., 2013). Datos similares obtiene Umadevi (2009), quien analizó la correspondencia entre la motivación de logro, la inteligencia emocional y la productividad académica en educación primaria, obteniendo un nexo positivo entre estos términos. En la investigación realizada por Higgs y Dulewicz (2014) se encuentra que la motivación de logro podría aumentar la inteligencia emocional, por lo que estos autores plantean la necesidad de impulsar estudios centrados en el bienestar. En esta línea, encontramos también los planteamientos de diferentes investigadores que hallan la existencia de una correlación entre la inteligencia emocional y motivación de logro (Kumar 2016; Naik y Kiran 2018; Katarzyna y Dariusz, 2019).

La inteligencia emocional también se relaciona con las metas vitales (Bastian et al., 2005). En concreto, Bastian et al. (2005) demostraron cómo la gestión de las emociones permite alcanzar las metas vitales. En su trabajo, estos autores descubrieron que la inteligencia emocional correlaciona con la satisfacción vital, el afrontamiento, la resolución de problemas y los bajos niveles de ansiedad. Dentro de esta línea, convendría destacar también el estudio de Mohanty et al. (2015), quienes evidenciaron que los individuos con inteligencia emocional cuentan con más ganas de realizar actividades que mejoren su vida, además se perciben como personas más sociables. En este trabajo se manifestó que los estudiantes que gestionan sus emociones tienen mejores relaciones interpersonales, se consideran autosuficientes y establecen mejores propósitos en su vida.

En síntesis, tal como se ha reflejado previamente, en el ámbito académico son necesarias más investigaciones que aborden en profundidad la función de la inteligencia emocional (Bisquerra, 2013). De manera más concreta, hemos mostrado que son escasos los trabajos centrados en la relación de esta variable con la motivación de logro y los propósitos vitales, así como la relación mediada entre estos términos. 


\section{Objetivos e hipótesis}

En este contexto enmarcamos nuestra investigación, donde pretendemos conocer qué factores emocionales y motivacionales pueden resultar relevantes para el logro de propósitos vitales. Para ello, se propone un estudio ex post facto (Montero y León, 2007) causal de corte transversal, cuyo objetivo general consiste en comprobar un modelo pragmático donde la inteligencia emocional desempeña un rol mediador entre la motivación de logro y las metas vitales de los jóvenes universitarios. A partir de la literatura, se propuso y se probó un modelo de mediación simple (ver figura 1), mediante el cual se estudió si la inteligencia emocional tiene un efecto mediador entre la motivación de logro y las metas vitales. En base a lo expuesto previamente, se plantean las siguientes hipótesis de trabajo:

Hipótesis 1. La motivación de logro mostrará relaciones estadísticamente significativas con la inteligencia emocional.

En lo concerciente a la segunda hipótesis, esta se respalda en el trabajo de Dulewicz y Higgs (2000), quienes impulsan una investigación donde concluyen que la motivación de logro puede estar relacionada con la inteligencia emocional. Y, también, se sustenta en el estudio de Higgs y Dulewicz (2014), cuyos resultados reflejan que la motivación de logro podría aumentar a la inteligencia emocional.

Hipótesis 2. La inteligencia emocional establecerá una relación estadísticamente significativa con las metas vitales.

Mohanty et al. (2015) demostraron la repercursión de la inteligencia emocional en el sentido vital. En concreto, hallaron que el crecimiento personal se encuentra relacionado con la curiosidad y el sentido vital y, también, que ambos constructos están mediados por la inteligencia emocional. En esta misma línea, Bastian et al. (2005) descubren que la inteligencia emocional es decisiva en el control del estado de ánimo lo que permitará el logro de las metas vitales.

Hipótesis 3. La motivación de logro establece una relación estadísticamente significativa con las metas vitales. 
Esta hipótesis se sustenta en los trabajos de Valdés et al. (2013) y Hardin et al. (2019). Los primeros autores demostraron que la motivación de logro orienta a los estudiantes en el cumplimiento de sus objetivos. Por su parte, Hardin et al. (2019) evidenciaron la existencia de una relación entre la motivación de logro y la permanencia académica.

Hipótesis 4. La inteligencia emocional mostrará una relación mediada estadísticamente significativa entre la relación de la motivación de logro y las metas vitales.

Esta hipótesis se enmarca en la investigación de Umadevi (2009), quien estudió la correspondencia entre la motivación de logro, la inteligencia emocional y el rendimiento académico en enseñanza básica (primaria). Los hallazgos obtenidos reflejan la existencia de una relación estadísticamente significativa y positiva entre estos términos.

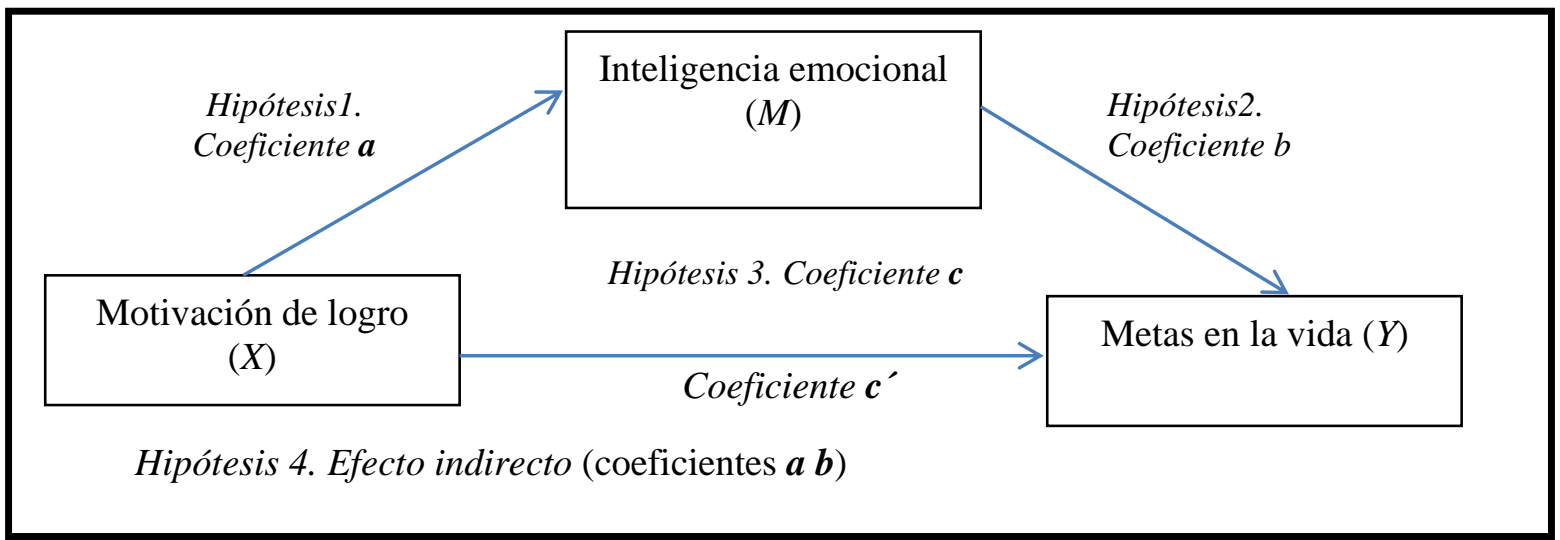

Figura 1. Modelo de mediación

\section{Método}

\section{Participantes}

Este trabajo es un estudio ex post facto causal de corte transversal, con un muestreo no probabilístico por conveniencia, donde participaron 311 estudiantes universitarios, de los cuales el $25 \%$ son hombres y el $75 \%$ mujeres. La edad de los participantes oscila entre los 17 hasta los 56 años, siendo la media de edad de 22 años ( $S d=4.85)$. Un 44\% eran estudiantes del grado en Psicología, seguido de un 27\% que estudiaban el grado en Trabajo Social, un $18 \%$ el grado en Educación Social y un 11\% grado en Educación Física. En este trabajo se siguieron las recomendaciones del comité bioético de la Universidad de Almería. 


\section{Instrumentos}

Trait Meta-Mood Scale (TMMS; Salovey et al., 1995). En este estudio se utilizó una versión española (Fernández-Berrocal, Extremera y Ramos, 2004), integrado por un total de 24 preguntas, con formato de respuesta tipo Likert de 5 alternativas de respuesta, siendo 1=Nada de acuerdo y 5=Totalmente de acuerdo. Mediante este cuestionario se evalúan tres dimensiones: La primera es la percepción o atención, o sea, ser capaz de sentir y expresar adecuadamente los sentimientos, la cual incluye un total de ocho preguntas, similares a la siguiente "presto mucha atención a los sentimientos" (alfa de Cronbach de .872). La segunda es la comprensión o claridad, esto es, comprender bien los estados emocionales, la cual está compuesta por ocho cuestiones similares al siguiente ítem: "tengo claros mis sentimientos" (alfa de Cronbach de .779). La tercera, que engloba otras ocho cuestiones, es la regulación o reparación, esto es, ser capaz de reparar correctamente los estados emocionales. En concreto, esta dimensión contiene preguntas del tipo "tengo mucha energía cuando me siento feliz" (alfa de Cronbach de .846). La consistencia general de la escala es de .859 .

Escala de motivación de logro (ML; Morales, 2006). Esta escala evalúa la motivación de logro a través de 16 items con formato de respuesta tipo Likert de 4 alternativas, siendo $1=$ Totalmente de acuerdo y 4=Totalmente en desacuerdo, un ejemplo de pregunta es el siguiente "prefiero tomar decisiones en grupo que aceptar yo toda la responsabilidad de las actividades del grupo". La consistencia interna de esta escala, obtenida con la prueba alfa de Cronbach, presenta un valor moderado (Rosenthal y Rubín, 2003) de .582.

Metas en la vida (Wilding y Andrews, 2006). En este trabajo se utilizó una versión española (Morales, 2010). Esta escala evalúa las orientaciones o metas en la vida a través de 11 preguntas con formato de respuesta tipo Likert de 6 alternativas, siendo 1=Nada importante y 6=Máxima importancia. Un ejemplo de pregunta sería la siguiente: "tener una carrera profesional muy gratificante”. La escala muestra una consistencia interna de .77.

\section{Procedimiento}

Se utilizó el programa SPSS para realizar los análisis estadísticos, en concreto, la macro PROCESS, con la que se llevó a cabo una comprobación de mediación con técnicas brootstrapping. Se hizo un estudio de mediación simple con una variable mediadora cuantitativa, una muestra de 10000 brootstrapping, modelo 4 y un nivel de confianza del 95\% (para construir los intervalos y determinar si el efecto indirecto es estadísticamente significativo). 
La Variable independiente (X) motivación de logro, variable dependiente (Y) metas en la vida y la variable mediadora (M) inteligencia emocional. Los criterios de Barón y Kenny (1986) fueron utilizados para realizar el análisis de mediación. Para ello, se calculó un modelo 4 final denominado del efecto mediador tanto de la relación con el factor mediador (inteligencia emocional) como la correspondencia directa entre los factores de motivación de logro y las metas vitales (ver Figura 1).

\section{Resultados}

\section{Análisis descriptivos y correlación de Pearson}

Como podemos observar en la tabla 1 se presentan los datos descriptivos y las correlaciones de las variables objeto de estudio. Tal como se aprecia en la misma existen correlaciones estadísticamente significativas entre las variables $(p \leq .01)$, siendo la relación de motivación de logro y metas en la vida la que desprende un peso más alto $(r=.165 ; p \leq .01)$.

Tabla 1. Análisis descriptivos y correlaciones de Pearson

\begin{tabular}{llllll}
\hline & $M$ & $S D$ & 1 & 2 & 3 \\
\hline 1.Motivación de logro & 2.94 & .23 & & & \\
2.Metas en la vida & 4.56 & .61 & $.165^{* *}$ & & \\
3.Inteligencia emocional & 3.31 & .57 & $.163^{* *}$ & $.159^{* *}$ & \\
\hline
\end{tabular}

\section{Modelo de efectos mediadores}

Según Barón y Kenny (1986) se establecen cuatro pasos para el análisis. El paso primero (coeficiente a), se trata de demostrar si la variable predictora $(\mathrm{X})$ está relacionada con la variable mediadora $(\mathrm{M}) \quad\left[R=.1648, \quad R^{2}=.0271, \quad M S E=.3191, \quad F=8.6233, \quad d f 1=1.000\right.$, $d f 2=309,0000, p \leq .0036]$. Nuestros datos reflejan un coeficiente de regresión no estandarizado con un valor positivo y estadísticamente significativo, luego existe una relación significativa y positiva entre la motivación de logro y la inteligencia emocional $(B=.403, S E=.137, t=2.94$, $p \leq .004, L L C I=.1328, U L C I=.672)$.

El segundo paso (coeficientes $b$ y $c^{c}$ ) centrado en demostrar la relación entre variables, por un lado, estudiar si la variable predictora $(\mathrm{X})$ está relacionada con la variable criterio $(\mathrm{Y})$ 
-coeficiente $c^{\prime}-\mathrm{y}$, por otro lado, conocer la vinculación de la variable mediadora (M) y la variable criterio (Y), coeficiente $b\left[R=.2124, R^{2}=.0451, M S E=.3599, F=7,278, d f 1=2.000\right.$, $d f 2=308,0000, p \leq .0008]$.

Los datos pertenecientes a la relación causal (motivación de logro) y efecto (metas vida) -coeficiente $c^{\prime}$ - muestran una asociación positiva y estadísticamente significativa $(B=.373$, $S E=.148, t=2.53, p \leq .01, L L C I=.083, U L C I=.633)$. En lo que respecta a la influencia de la variable mediadora (inteligencia emocional) sobre la variable dependiente (metas en la vida), coeficiente $b$, observamos que la relación es estadísticamente significativa $(B=.1454$, $S E=.0604, t=2.4073, p \leq .0167, L L C I=.0266, U L C I=.2643)$. En síntesis, los efectos de ambos coeficientes ( $\mathrm{b} \mathrm{c}^{\prime}$ ) sobre la variable dependiente son positivos y estadísticamente significativos.

El tercer paso, relacionado con el efecto total, es un análisis de regresión con solo un predictor (coeficiente c) $\left[R=.1648, \quad R^{2}=.0272, \quad M S E=.3655, \quad F=8.6233, \quad d f 1=1.000\right.$, $d f 2=309.0000, p \leq .0036]$, cuyo resultado es positivo y estadísticamente significativo $(B=.4310$, $S E=.1467, t=2.9373, p \leq .0036, L L C C=1423, U L C I=.7198)$.

En el cuarto paso se pretende demostrar si la intensidad de la relación de la variable independiente $(\mathrm{X})$ con la variable dependiente $(\mathrm{Y})$ es estadísticamente significativa con un peso menor cuando interviene la variable mediadora (M), efecto indirecto bootstrapping ab. Tal como reflejan los datos (ver tabla 2), el tamaño del coeficiente es de .0585 , con una desviación estándar, construido con el método de bootstrapping, de .0340 y un intervalo de confianza con un límite superior de .0096 y un límite inferior de .1486. Tal como se aprecia en dicho intervalo, el efecto es estadísticamente significativo con signo positivo.

Tabla 2. Efectos totales, directos e indirectos

\begin{tabular}{lllllll}
\hline & Efectos & $S E$ & $t$ & $p$ & LLCI & ULCI \\
\hline Efectos de X en $Y$ & .43 & .14 & 2.93 & .0036 & .1423 & .7198 \\
Efectos directos & .37 & .14 & 2.52 & .0121 & .0820 & .6630 \\
de X en $Y$ & & & & & & \\
Efectos indirectos & .058 & .03 & & & .0097 & .1486 \\
de X en $Y$ & & & & & & \\
\hline
\end{tabular}

$\mathrm{X}=$ Motivación de logro; $\mathrm{Y}=$ Metas en la vida 
En síntesis, con estos datos podríamos decir que el efecto indirecto es estadísticamente significativo (CI 95\%). Podemos concluir que la motivación de logro genera una mayor posibilidad de plantearse metas vitales. Además, el efecto indirecto nos indica que se explica por la actuación de una variable mediadora (inteligencia emocional). De tal manera que la motivación de logro activa la inteligencia emocional y la inteligencia emocional influye en las metas marcadas en la vida (ver figura 2). Por consiguiente, la inteligencia emocional es mediadora de esta relación, es decir, tener motivación de logro se relaciona positivamente y significativamente con las metas vitales de modo indirecto a través de su efecto con la inteligencia emocional.

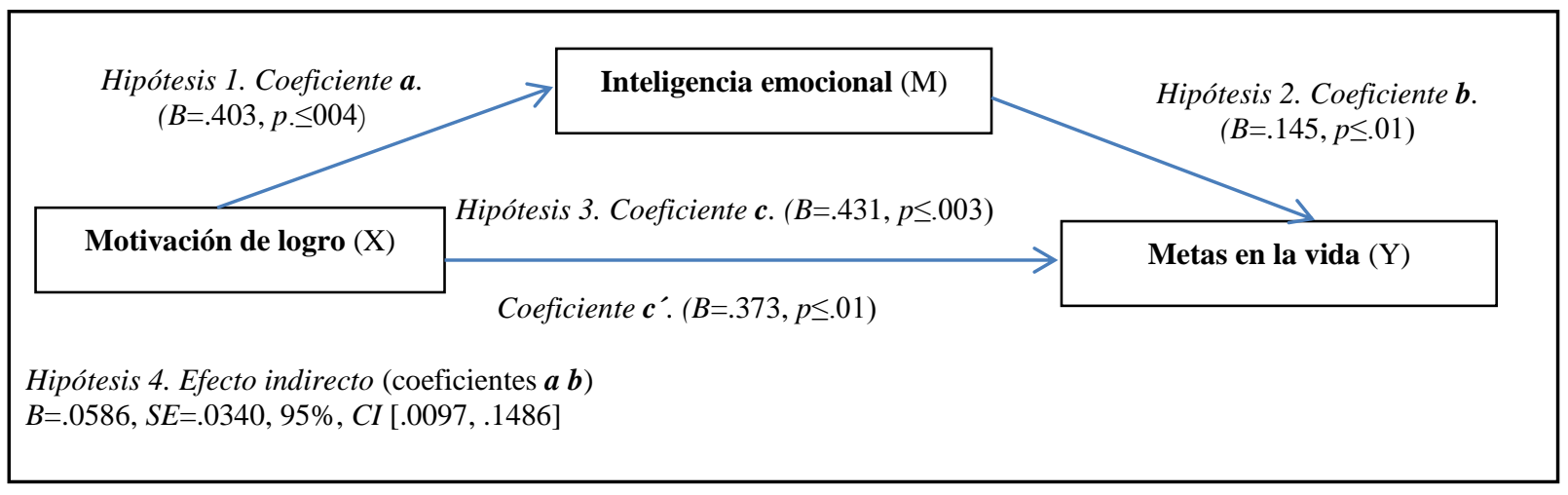

Figura 2. Estimaciones estandarizadas para el Modelo de mediación

\section{Discusión y Conclusiones}

El propósito de este estudio se centraba en analizar la relación entre la motivación de logro, la inteligencia emocional y las metas vitales, particularmente conocer el efecto de medicación de la inteligencia emocional entre la motivación de logro y las metas vitales. Los resultados son estadísticamente significativos y positivos en las relaciones entre estas variables. Con estos hallazgos, nuestra investigación podría ofrecer una contribución innovadora, al destacar el papel amortiguador que la inteligencia emocional presenta en la influencia de motivación de logro (Dulewicz y Higgs, 2000; Magnano et al., 2016; Umadevi, 2009) y metas vitales (Roy et al., 2013).

En lo concerniente a la primera hipótesis, encontramos que la motivación de logro se relaciona significativamente y positivamente con la inteligencia emocional. A diferencia de los resultados obtenidos por Roy et al. (2013), nuestro trabajo confirma una relación estadísticamente significativa con un peso moderado entre la motivación de logro y la inteligencia 
emocional, estos hallazgos concuerdan con los obtenidos en el estudio de Higgs y Dulewicz (2014). El trabajo de Dulewicz y Higgs (2000) nos lleva a plantear que aprender a gestionar las emociones podría verse afectado por la motivación de logro, dicho con otras palabras, la necesidad o el impulso por adquirir hábitos, destrezas y habilidades podría llevar a los estudiantes a aprender a gestionar sus emociones (Higgs y Dulewicz, 2014), es decir, a dominar sus propias emociones.

En lo que respecta a la segunda hipótesis, descubrimos que la inteligencia emocional influye de manera positiva y estadísticamente significativa en las metas vitales. Estos datos revelan que el control de las emociones puede resultar positivo para alcanzar las metas y ser perseverantes. Nuestros hallazgos coinciden con los obtenidos por Mohanty et al. (2015) que demostraron la repercursión de la inteligencia emocional en el sentido vital, esto es, el crecimiento personal. En línea con nuestros resultados destacamos la investigación realizada por Chowdhury et al. (2007) y otros autores (Brackett y Mayer, 2003; Brackett et al., 2006; Lunda et al. 2010), que evidencian la relación estadísticamente significativa entre la inteligencia emocional y la satisfacción con la vida.

En lo que respecta a la tercera hipótesis se confirma que la motivación de logro se realaciona positiva y significativamente con las metas vitales. Estos datos coinciden con los establecidos por distintos autores (Valdés et al., 2013; Hardin et al., 2019). Estos hallazgos indican que el impulso por mejorar hábitos, destrezas y habilidades podría afectar a las metas que los estudiantes se marquen en su vida.

Por último, en la cuarta hipótesis, se verificó que la inteligencia emocional es una variable mediadora entre la motivación de logro y las metas vitales. Pese a todo, debido al escaso número de estudios previos destinados a comprobar el modelo teórico propuesto, resulta complicado contrastar nuestros resultados con investigaciones similares. Aún así, quisiéramos destacar el trabajo de Umadevi (2009), quien estudió la correspondencia entre la motivación de logro, la inteligencia emocional y el rendimiento académico en educación primaria. En este sentido, el hecho de que la relación entre la motivación de logro y los propósitos vitales se encuentre mediada por la inteligencia emocional viene a confirmar los planteamientos de que trabajar las competencias motivacionales podrían ejercer mejoras en el desarrollo emocional (Pekrun, 1992) y podrían mejorar los planteamientos sociales al ser perseverantes con el cumplimiento de metas y reduciendo la probabilidad de comportamientos problemáticos (Durlak 
et al., 2011). En síntesis, entre nuestros hallazgos principales podríamos destacar que, aunque no existan motivadores externos, los estudiantes que tienen motivación de logro podría resultarle más fácil gestionar sus emociones, la cual, a su vez, podría contribuir a mantener la intención de llegar a lograr las metas vitales propuestas. Tal como ha quedado demostrado, resulta, por tanto, fundamental identificar y promover aspectos motivacionales que contribuyan a la adquisición de habilidades emocionales que ayuden a afrontar satisfactoriamente los retos vitales (Seligman, 1999).

\section{Limitaciones prácticas}

Este trabajo presenta algunas limitaciones, la primera está relacionada con el método utilizado para obtener los datos. En este estudio se han usado sólo herramientas cuantitativas y al tratarse del tema emocional quizás convendría emplear metodogía mixta en el mismo trabajo, es decir, cuestionarios con métodos cualitativos, como por ejemplo un diario de emociones. La segunda está vinculada con el tipo de estudio, ya que se trata de un trabajo transversal, aplicado en un momento temporal. A este respecto parece necesario impulsar investigaciones de corte longitudinal con la finalidad de obtener una mayor validación en las relaciones causales establecidas en el modelo. La tercera está asociada al perfil muestral, en concreto, en este aspecto queremos destacar que la muestra está constituida principalmente por mujeres, lo que podría suponer un condicionante en los resultados obtenidos. La cuarta, el tipo de muestreo utilizado puede resultar útil para la exploración de modelos teóricos, aunque deberíamos ser cautelosos con la divulgación de los resultados. De hecho, se requieren investigaciones futuras con una muestra más amplia y representativa para establecer si estos resultados son replicables. La quinta está relacionada con la consistencia desprendida en la escala de motivación de logro. Tal como se refleja en el apartado de instrumento el valor alcanzado es bajo, pese a que como indica Rosenthal (1994, citado en García, 2005), este valor puede ser considerado según el propósito de la investigación, es decir, si el objetivo es comprobar la viabilidad de un modelo teórico puede aceptarse un valor de consistencia bajo. En síntesis, recomendamos impulsar más investigaciones sobre esta temática teniendo en cuenta las limitaciones citadas en el presente trabajo para comprobar si estas relaciones se mantienen.

\section{Implicaciones prácticas}

En lo que respecta a las implicaciones prácticas podríamos establecer que los estudiantes con mayor motivación de logro gestionan mejor sus emociones y esto genera consecuencias en sus metas vitales. En este sentido, según apuntan los datos, si en el ámbito académico 
se pretende conseguir buenos resultados académicos y, también, que los estudiantes sean persistentes en el logro de metas vitales convendría trabajar tanto la motivación de logro como la gestión de las emociones. En lo que respecta a la inteligencia emocional, tal como señala Bisquerra (2003) la educación emocional es un componente importante que no suele ser atendido en el ámbito educativo. Pues bien, en base a estos datos deberíamos plantearnos la posibilidad (o la necesidad) de incluir en el currículum actividades que contribuyan a la gestión de las emociones. En este sentido, con esta forma de intervención, se lograría conseguir un desarrollo integral de las personas, es decir, se conseguiría una mejora personal y social (Bisquerra, 2003). Aún más, esto se ve justificado cuando descubrimos que la inteligencia emocional influye en los propósitos vitales, en este sentido, parece que trabajar la inteligencia emocional capacitaría más a los estudiantes para afrontar exitosamente los retos vitales. De la misma manera, para conseguir satisfactoriamente las metas vitales es fundamental tener motivación de logro. En nuestro trabajo se evidencia también que la motivación de logro puede resultar básica tanto para el establecimiento de las metas vitales como para aprender a controlar las emociones.

Por último, quisiéramos destacar que con este trabajo no sólo se pone en evidencia la importancia de la inteligencia emocional y la motivación de logro en el ámbito académico, sino que, además, se comprueba un modelo teórico causal, con el que se ofrece una visión más específica de la relación existente entre estos términos, siendo la gestión emocional una variable mediadora. Por ende, queremos enfatizar que, tal como apuntan algunos autores (Cohen, 2006; Durlak et al., 2011; Casey y Paulson, 2011; Kress y Elias, 2006; Weare y Nind, 2011; Zins et al., 2004), los docentes pueden contribuir/ayudar proactivamente para que los estudiantes sean perseverantes en el logro de sus metas vitales mediante la enseñanza de habilidades motivacionales y emocionales.

\section{Referencias}

Baron, R. M. y Kenny, D. A. (1986). The moderator-mediator variable distinction in social psychological research: Conceptual, strategic, and statistical considerations. Journal of Personality and Social Psychology, 51, 1173-1182. https://doi.org/10.1037/0022-3514.51.6.1173.

Bastian, V., Burns, N. y Nettelbeck, T. (2005). Emotional intelligence predicts life skills, but not as well as personality and cognitive abilities. Personality and Individual Differences, 39,11351145. 
Brackett, M.A. y Mayer, J.D. (2003). Emotional Intelligence and the Prediction of Behaviour. Personality and Social Psychology Bulletin, 29, 1147-1158.

Brackett, M.A., Lopes, P., Ivcevic, Z., Mayer, J.D. y Salovey, P. (2006). Integrating Emotion and Cognition: The Role of Emotional Intelligence. En Motivation, Emotion and Cognition: Integrating Perspectives on Intellectual Functioning, eds. D. Dai and R. Sternberg, Mahwah, NJ: Erlbaum.

CASEL GUIDE (2013). Effective Social and Emotional Learning Programs. EEUU, Arlington: KSAPlus Communications, Inc.

Chowdhury, M.S., Mohammed S. y Shahabuddin, A.M. (2007). Self Efficacy, Motivation and their Relationship to Academic Performance of Bangladesh College Students. College Quarterly, $10,1-9$.

Cooper, R. K. y Sawaf, A. (1997). Executive EQ: Emotional Intelligence in Leadership and Organizations. New York: Advanced Intelligence Technologies, LLC.

Dulewicz, V. y Higgs, M. (1999). EIQ User Manual. NFER-Nelson, Windsor.

Dulewicz, V. y Higgs, M. (2000). Emotional Intelligence. A review and evaluation study. Journal of Managerial Psychology, 15(4), 341-372. https://doi.org/10.1108/02683940010330993.

Durlak, J. A., Weissberg, R. P., Dymnicki, A. B., Taylor, R. D. y Schellinger, K. B. (2011). The impact of enhancing students' social and emotional learning: A meta-analysis of school-based universal interventions. Child Development, 82(1), 405-432. doi: 10.1111/j.14678624.2010.01564.x.

Edmund, J. (2011). Sea un líder influyente. Michigan: Portavoz.

Fernández, L. (2008). Estilos de aprendizaje, motivación de logro y satisfacción en los contextos online. Revista de estilos de aprendizaje, 2(1), 153-169.

Fernández-Berrocal, P., Extremera, N., y Ramos, N. (2004). Validity and reliability of the Spanish modified version of the Trait Meta-mood Scale. Psychological Reports, 94, 751-755.

García, H. (2006). La medición en ciencias sociales y en Psicología. En Landeros, R. y Gonzalez, M. (Comp.), Estadística con SPSS y metodología de la investigación. México: Trillas.

García, F. y Domenech, F. (1997). Motivación, aprendizaje y rendimiento escolar. Revista Electrónica de Motivación y Emoción, 1, 55-65.

Gardner, H. (1993). Multiple Intelligences: The Theory in Practice. Nueva York: Basic Books.

Goleman, D. (1995). Emotional intelligence. Nueva Cork, NY: Bantum Books.

Higgs, M. y Dulewicz, V. (1999). Can Emotional Intelligence be measured and developed? Leadership and Organization Development, 20(5), 242-252. doi:10.1108/01437739910287117

Higgs, M. y Dulewicz, V. (2014). Antecedents of well-being: a study to examine the extent to which personality and emotional intelligence contribute to well-being. The International Journal of Human Resource Management, 25(5), 718-735. DOI: 10.1080/09585192.2013.81525. 
Katarzyna, R. y Dariusz, G. (2019). Achievement Motivation and Emotional Intelligence in Elite Female and Male Wrestlers. Journal of Martial Arts Anthropology, 20(1), 14-22 DOI: 10.14589/ido.20.1.2

Kumar K. (2016). Emotional intelligence and achievement motivation: a correlation study. Indian Journal of Health and Wellbeing, 7(5), 546-549.

Lozano, S. y Repetto, E. (2007). Las dificultades en el proceso de decisión vocacional en relación con: el género, el curso académico y los intereses profesionales. Revista de Orientación y Psicopedagogía, 18(1), 5-16. https://doi.org/10.5944/reop.vol.18.num.1.2007.11293.

Lunda, J.M.A., Martos, M.P. y López-Zafra, E. (2010). Emotional Intelligence and Personality Traits as Predictors of Psychological Well-Being in Spanish Undergraduates. Social Behaviour and Personality, 38(6), 783-793.

Magnano, P., Crasparo, G. y Paolillo, A. (2016). Resilience and Emotional Intell gence: which role in achievement motivation. International Journal of Psychology Resesarch, 9(1), 9-20 .

Mayer, J. D., Salovey, P. y Caruso, D. (2004). Models of Emotional Intelligence. En R.J. Sternberg (Ed.), Handbook of Intelligence (2nd Ed.). Cambridge: Cambridge University Press.

Mohanty, A., Pradhan, R.K. y Jena, L.K. (2015). Curiosity and Meaning of life leading towards Personal Growth: The role of Emotional Intelligence. Journal of the Indian Academy of Applied Psychology, 41(2), 1-35.

Morales, P. (2006). Medición de actitudes en Psicología y Educación; construcción de escalas y problemas metodológicos. Madrid: Universidad Pontificia Comillas.

Morales, P. (2010). Cuestionario y escalas. Madrid: Facultad de Ciencias Humanas y Sociales. Universidad Pontificia Comillas (versión revisada 19 de Febrero).

Nathanson, L., Rivers, S., Flynn, L. y Brackett, M. (2016). Creating Emotionally Intelligent Schools with RULER. Emotion Review, 8(4), 1-6. DOI: 10.1177/1754073916650495 er.sagepub.com

Naik D. y Kiran D.A. (2018). Emotional intelligence and achievement motivation among college students. Indian Journal of Health and Well-being, 9(1), 86-88.

Ocampo, C., Ferro, S. y Ojea, M. (2001). Psicoloxía vocacional e programas de orientación profesional: aspectos conceptuais básicos. Revista Galego-Portuguesa de Psicoloxía e Educación, 7, $33-42$.

Pekrun, R., Goetz, T. y Titz, W. (2002). Academic Emotions in Students' Self-Regulated Learning and Achievement: A Program of Qualitative and Quantitative Research. Educational Psychologist, 37(2), 91-106. https://doi.org/10.1207/S15326985EP3702_4

Pekrun, R. (1992). The Impact of Emotions on Learning and Achievement: Towards a Theory of Cognitive/Motivational Mediators. Applied Psychology: An International Review, 41 (4), 359-376. DOI: 10.1111/j.1464-0597.1992.tb00712.x.

Rosenthal, J.A. (1994). Reliability and social work research. Social Work Research, 18(2), 115-121. http://doi.org/10.1093/swr/18.2.115 
Rosenthal, R. y Rubin, D. B. (2003). Requivalent: A simple effect size indicator. Psychological Methods, 8(4), 492- 496. doi:10.1037/1082-989X.8.4.492.

Roy, B., Sinha, R. y Suman, S. (2013). Emotional intelligence and academic achievement motivation among adolescents: A relationship study. Journal of Arts, Science y Commerce, 4(2), 126-130. DOI: 10.1177/0961000618790629.

Ryff, C. (1989). Happiness is everything, or is it? Explorations on the meaning of psychological wellbeing. Journal of Personality and Social Psychology, 57, 1069-1081.

Salovey, P. y Mayer, J. (1990). Emotional intelligence. Imagination, Cognition and Personality, 9 (3), 185-211. doi:0.2190/DUGG-P24E-52WK-6CDG.

Salovey, P., Mayer, J. D., Goldman, S. L., Turvey, C. y Palfai, T. P. (1995). Emotional attention, clarity and repair: exploring emotional intelligence using the Trait Meta Mood Scale. In J. W. Pennebaker (Ed.), Emotion, disclosure and health (pp. 125-154). Washington, DC: American Psychological Association.

Seligman, M.E.P. (1999). The president's address. American Psychologist, 54, 559-532.

Takeuchi, H., Taki, Y., Nouchi, R., Sekiguchi, A., Makoto, C., Yokoyama, R., Lizuka, K. y Kawashima, R. (2014). Regional gray matter density in associated with achievement motivation: Evidence form voxel-based morphometry. Brain Structure Function, 219, 71-83. doi: 10.1007/s00429-012-0485-3. Epub 2012 Dec 5.

Umadevi, M. R. (2009). Relationship between Emotional Intelligence, Achievement Motivation and Academic Achievement. Edutracks, 8(12), 31-35.

Valdés, A., Sánchez, P. y Yañez, A. (2013). Perfiles de estudiantes mexicanos con aptitudes intelectuales sobresalientes. Acta Colombiana de Psicología, 16(1), 25-33.

Van Damme S., Van Ryckeghem D.M., Wyffels F., Van Hulle L. y Crombez G. (2012). No pain no gain? Pursuing a competing goal inhibits avoidance behavior. Pain, 153, 800-804. doi: 10.1016/j.pain.2011.12.015. Epub 2012 Feb 1.

Wilding, J. y Andrews, B. (2006). Life goals, approaches to study and performance in an undergraduate cohort. British Journal of Educational Psychology, 76 (1), 171-182.

Yazici, H. Syis, S. y Altum, F. (2011). Emotional intelligence and self-efficacy beliefs as predictors of academia achievement among high school students. Procedia: Social and Behavioral Science, 15, 2319-2323. doi:10.1016/j.sbspro.2011.04.100.

Recibido: 23-06-2020

Acceptado: 15-12-2020 